\title{
Identification of Protein Changes in the Urine of Hypothyroid Patients Treated with Thyroxine Using Proteomics Approach
}

\author{
Afshan Masood ${ }^{1}$, Hicham Benabdelkamel ${ }^{1 *}$, Anwar A Jammah², Aishah A Ekhzaimy², \\ Assim A Alfadda ${ }^{1,2}$ \\ ${ }^{1}$ Proteomics Resource Unit, Obesity Research Center, College of Medicine, King Saud University, Riyadh, \\ Saudi Arabia \\ ${ }^{2}$ Department of Medicine, College of Medicine, King Saud University, Riyadh, Saudi Arabia. \\ * Correspondence: \\ Hicham Benabdelkamel \\ Proteomics Resource Unit, Obesity Research Center, College of Medicine, King Saud University, Riyadh, \\ Saudi Arabia \\ Email: hbenabdelkamel@ksu.edu.sa
}




\section{SUPPORTING INFORMATAION}

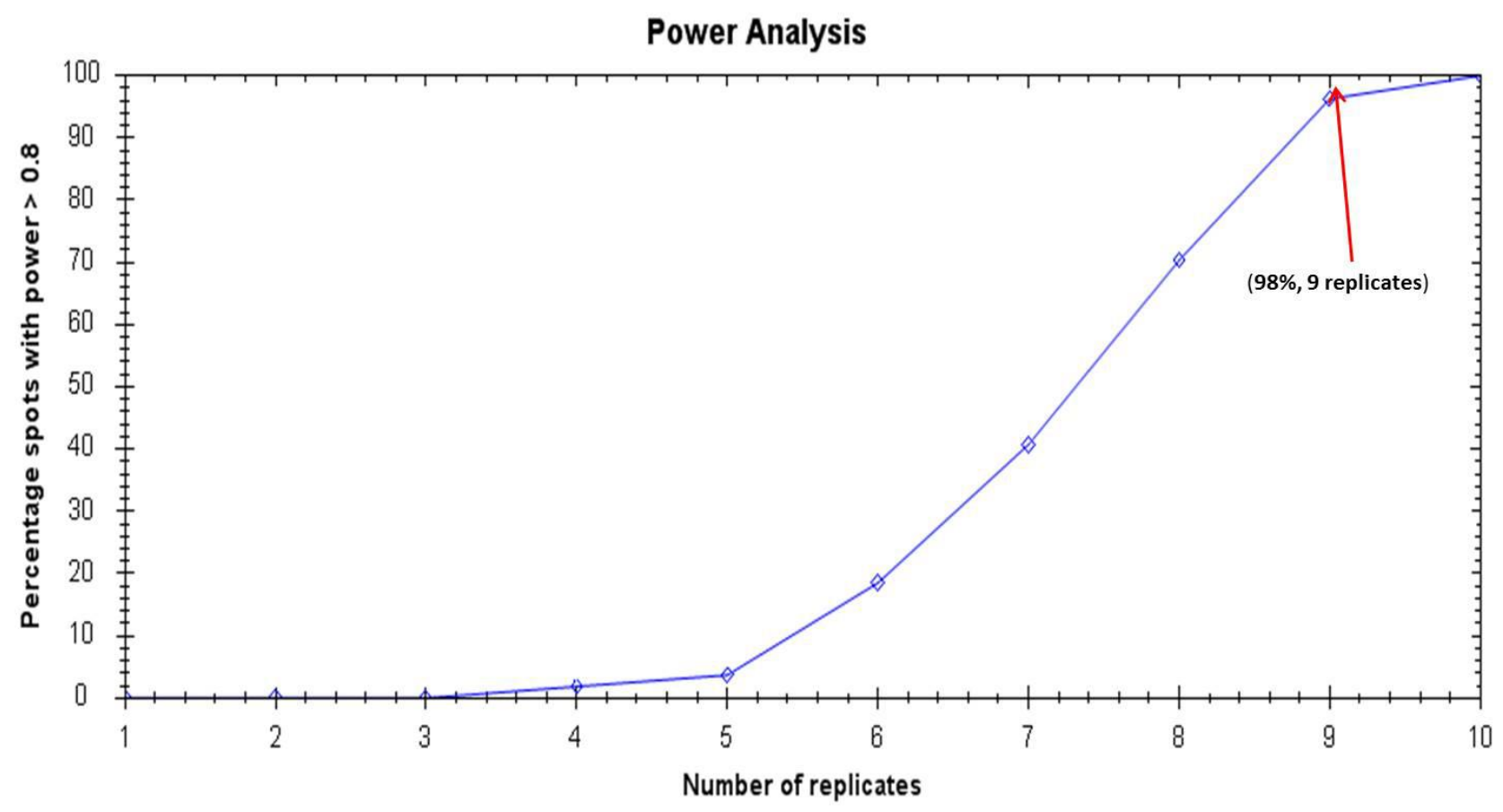

Sample Size (number of required biological replicates)

Figure S1: Power calculation for determination of the minimum number of required biological variants for 2-DIGE analysis. The power curve was used to calculate the sample size required to find significant difference with a fold-change of $\geq 1.5$ between two paired groups at $98 \%$ power and $\mathrm{p}$-value $\leq 0.05$ 


\begin{tabular}{|c|c|c|}
\hline ID & $\begin{array}{l}\text { Associated Network } \\
\text { Functions }\end{array}$ & Score \\
\hline 1 & $\begin{array}{l}\text { Amino Acid } \\
\text { Metabolism, Molecular } \\
\text { Transport, Small } \\
\text { Molecule Biochemistry }\end{array}$ & 26 \\
\hline 2 & $\begin{array}{l}\text { Gastrointestinal } \\
\text { Disease, Organismal } \\
\text { Injury and } \\
\text { Abnormalities, } \\
\text { Connective Tissue } \\
\text { Disorders }\end{array}$ & 26 \\
\hline 3 & $\begin{array}{l}\text { Cardiovascular System } \\
\text { Development and } \\
\text { Function, Organ } \\
\text { Development, Cell } \\
\text { Death and Survival }\end{array}$ & 20 \\
\hline
\end{tabular}

Top Canonical Pathways

\begin{tabular}{lcc} 
Name & p-value & Overlap \\
\hline LXR/RXR Activation & $6.49 \mathrm{E}-07$ & $4.1 \% 5 / 121$ \\
FXR/RXR Activation & $7.93 \mathrm{E}-07$ & $4.0 \% 5 / 126$ \\
Acute Phase Response Signaling & $4.47 \mathrm{E}-06$ & $2.8 \% 5 / 179$ \\
Clathrin-mediated Endocytosis Signaling & $2.38 \mathrm{E}-03$ & $1.6 \% 3 / 193$ \\
Coenzyme A Biosynthesis & $4.15 \mathrm{E}-03$ & $33.3 \% 1 / 3$
\end{tabular}

Figure S2: The figure shows the different canonical pathways obtained from IPA functional analysis. 
Table S1: Experimental design: 18 samples run on 9 2D-PAGE gels, samples were labeled randomly with $\mathrm{Cy} 3$ and $\mathrm{Cy} 5$, and a pooled sample was used as an internal standard and was stained with Cy2

\begin{tabular}{|c|c|c|c|}
\hline Gel & Cy3 & Cy5 & Cy2 \\
\hline 1 & $\begin{array}{c}1 \\
\text { (Euthyroid) }\end{array}$ & $\begin{array}{c}11 \\
\text { (hypothyroid) }\end{array}$ & Pooled sample \\
\hline 2 & $\begin{array}{c}12 \\
\text { (hypothyroid) }\end{array}$ & $\begin{array}{c}2 \\
\text { (Euthyroid) }\end{array}$ & Pooled sample \\
\hline 3 & $\begin{array}{c}\mathbf{5} \\
\text { (Euthyroid) }\end{array}$ & $\begin{array}{c}13 \\
\text { (hypothyroid) }\end{array}$ & Pooled sample \\
\hline 4 & $\begin{array}{c}14 \\
\text { (hypothyroid) }\end{array}$ & $\begin{array}{c}3 \\
\text { (Euthyroid) }\end{array}$ & Pooled sample \\
\hline 6 & $\begin{array}{c}16 \\
\text { (hypothyroid) }\end{array}$ & $\begin{array}{c}6 \\
\text { (Euthyroid) }\end{array}$ & Pooled sample \\
\hline 7 & $\begin{array}{c}7 \\
\text { (Euthyroid) } \\
\end{array}$ & $\begin{array}{c}17 \\
\text { (hypothyroid) }\end{array}$ & Pooled sample \\
\hline 8 & $\begin{array}{c}18 \\
\text { (hypothyroid) }\end{array}$ & $\begin{array}{c}8 \\
\text { (Euthyroid) }\end{array}$ & Pooled sample \\
\hline 9 & $\begin{array}{c}9 \\
\text { (Euthyroid) }\end{array}$ & $\begin{array}{c}19 \\
\text { (hypothyroid) }\end{array}$ & Pooled sample \\
\hline
\end{tabular}


Table S2: Mass spectrometry list of significant differentially abundant proteins between Hypo and Euth identified in urine samples, using 2D-DIGE with. Protein name, accession number, Mascot score, MS \% coverage, protein MW and pI values according to

Uniprot database are listed.

\begin{tabular}{|c|c|c|c|c|c|c|c|c|}
\hline SI no: & Spot No & Accession $\mathrm{No}^{\mathrm{b}}$ & Protein Name & MASCOT ID & $\mathbf{P i}^{\mathbf{c}}$ & $M W^{d}$ & Cov\% & Score ${ }^{e}$ \\
\hline 1. & 578 & P02768 & Serum albumin & ALBU_HUMAN & 5.92 & 71317 & 37 & 81 \\
\hline 2. & 376 & P02768 & Serum albumin & ALBU_HUMAN & 5.92 & 71317 & 41 & 132 \\
\hline 3. & 918 & Q8IZU9 & Kin of IRRE-like protein 3 & KIRR3_HUMAN & 6.46 & 86227 & 17 & 57 \\
\hline 4. & 356 & P02768 & Serum albumin & ALBU_HUMAN & 5.92 & 71317 & 40 & 121 \\
\hline 5. & 644 & Q96CD2 & $\begin{array}{l}\text { Phosphopantothenoylcysteine } \\
\text { decarboxylase }\end{array}$ & COAC_HUMAN & 5.72 & 22741 & 42 & 64 \\
\hline 6. & 110 & Q8NFX7 & Syntaxin-binding protein 6 & STXB6_HUMAN & 9.19 & 23824 & 39 & 57 \\
\hline 7. & 481 & 095395 & $\begin{array}{l}\text { Beta-1,3-galactosyl-O-glycosyl- } \\
\text { glycoprotein beta-1,6- } \mathrm{N} \text { - } \\
\text { acetylglucosaminyltransferase } 3\end{array}$ & GCNT3_HUMAN & 8.53 & 51572 & 19 & 57 \\
\hline
\end{tabular}




\begin{tabular}{|c|c|c|c|c|c|c|c|c|}
\hline 8. & 450 & 095395 & $\begin{array}{l}\text { Beta-1,3-galactosyl-O-glycosyl- } \\
\text { glycoprotein beta-1,6-N- } \\
\text { acetylglucosaminyltransferase } 3\end{array}$ & GCNT3_HUMAN & 8.53 & 51572 & 27 & 58 \\
\hline 9. & 808 & Q9HBZ2 & $\begin{array}{c}\text { Aryl hydrocarbon receptor nuclear } \\
\text { translocator } 2\end{array}$ & ARNT2_HUMAN & 6.25 & 79268 & 23 & 58 \\
\hline 10. & 503 & P01112 & GTPase HRas & RASH_HUMAN & 5.16 & 21627 & 38 & 58 \\
\hline 11. & 700 & 075409 & Huntingtin-interacting protein M & HYPM_HUMAN & 4.93 & 13547 & 65 & 60 \\
\hline 12. & 117 & P02787 & Serotransferrin & TRFE_HUMAN & 6.81 & 79280 & 43 & 158 \\
\hline 13. & 170 & P02768 & Serum albumin & ALBU_HUMAN & 5.92 & 71317 & 35 & 81 \\
\hline 14. & 742 & P21281 & $\begin{array}{l}\text { V-type proton ATPase subunit B, brain } \\
\text { isoform }\end{array}$ & VATB2_HUMAN & 5.57 & 56807 & 20 & 78 \\
\hline 15. & 743 & 095395 & $\begin{array}{c}\text { Beta-1,3-galactosyl-O-glycosyl- } \\
\text { glycoprotein beta-1,6- } \mathrm{N} \text { - } \\
\text { acetylglucosaminyltransferase } 3\end{array}$ & GCNT3_HUMAN & 8.53 & 51572 & 25 & 71 \\
\hline 16. & 735 & Q5T197 & E3 ubiquitin-protein ligase DCST1 & DCST1_HUMAN & 9.28 & 82142 & 23 & 58 \\
\hline 17. & 750 & P07339 & Cathepsin D & CATD_HUMAN & 6.10 & 45037 & 27 & 63 \\
\hline 18. & 819 & Q8N806 & $\begin{array}{l}\text { Putative E3 ubiquitin-protein ligase } \\
\text { UBR7 }\end{array}$ & UBR7_HUMAN & 4.70 & 49336 & 21 & 58 \\
\hline 19. & 509 & P13645 & Keratin, type I cytoskeletal 10 & K1C10_HUMAN & 5.13 & 59020 & 30 & 75 \\
\hline
\end{tabular}




\begin{tabular}{|c|c|c|c|c|c|c|c|c|}
\hline 20. & 498 & P01042 & Kininogen-1 & KNG1_HUMAN & 6.34 & 72556 & 21 & 57 \\
\hline 21. & 655 & Q8NF91 & Nesprin-1 & SYNE1_HUMAN & 5.38 & 1017069 & 6 & 61 \\
\hline 22. & 648 & Q9UQ35 & $\begin{array}{c}\text { Serine/arginine repetitive matrix } \\
\text { protein } 2\end{array}$ & SRRM2_HUMAN & 12.05 & 300179 & 10 & 63 \\
\hline 23. & 435 & Q8NF91 & Nesprin-1 & SYNE1_HUMAN & 5.38 & 1017069 & 4 & 64 \\
\hline 24. & 493 & Q8NF91 & Nesprin-1 & SYNE1_HUMAN & 5.38 & 1017069 & 4 & 66 \\
\hline 25. & 547 & Q6FIF0 & AN1-type zinc finger protein 6 & ZFAN6_HUMAN & 6.87 & 23168 & 27 & 56 \\
\hline 26. & 752 & Q96PF1 & $\begin{array}{l}\text { Protein-glutamine gamma- } \\
\text { glutamyltransferase } Z\end{array}$ & TGM7_HUMAN & 6.54 & 80575 & 17 & 58 \\
\hline 27. & 105 & A6NJ69 & IgA-inducing protein homolog & IGIP_HUMAN & 9.13 & 6161 & 98 & 58 \\
\hline 28. & 104 & Q9UP79 & $\begin{array}{l}\text { A disintegrin and metalloproteinase } \\
\text { with thrombospondin motifs } 8\end{array}$ & ATS8_HUMAN & 5.78 & 98890 & 17 & 59 \\
\hline 29. & 730 & 015212 & Prefoldin subunit 6 & PFD6_HUMAN & 8.83 & 14574 & 35 & 57 \\
\hline 30. & 608 & Q8N7C3 & $\begin{array}{l}\text { Probable E3 ubiquitin-protein ligase } \\
\text { TRIML2 }\end{array}$ & TRIMM_HUMAN & 5.52 & 44525 & 18 & 57 \\
\hline 31. & 728 & Q6FIF0 & AN1-type zinc finger protein 6 & ZFAN6_HUMAN & 6.87 & 23168 & 24 & 57 \\
\hline
\end{tabular}




\begin{tabular}{|c|c|c|c|c|c|c|c|c|}
\hline 32. & 828 & Q6FIF0 & AN1-type zinc finger protein 6 & ZFAN6_HUMAN & 6.87 & 23168 & 27 & 61 \\
\hline 33. & 698 & 13647 & Keratin, type II cytoskeletal 5 & K2C5_HUMAN & 7.59 & 62568 & 20 & 70 \\
\hline 34. & 734 & P02760 & Protein AMBP & AMBP_HUMAN & 3.95 & 39886 & 23 & 70 \\
\hline 35. & 702 & Q12907 & $\begin{array}{l}\text { Vesicular integral-membrane protein } \\
\text { VIP36 }\end{array}$ & LMAN2_HUMAN & 6.46 & 40545 & 30 & 75 \\
\hline 36. & 756 & Q96KA5 & $\begin{array}{l}\text { Cleft lip and palate transmembrane } \\
\text { protein 1-like protein }\end{array}$ & CLP1L_HUMAN & 8.71 & 62531 & 25 & 57 \\
\hline 37. & 726 & P02760 & Protein AMBP & AMBP_HUMAN & 3.95 & 39886 & 28 & 65 \\
\hline 38. & 718 & A8K0R7 & Zinc finger protein 839 & ZN839_HUMAN & 6.11 & 88570 & 21 & 75 \\
\hline 39. & 741 & P02760 & Protein AMBP & AMBP_HUMAN & 3.95 & 39886 & 23 & 60 \\
\hline 40. & 802 & Q93099 & Homogentisate 1,2-dioxygenase & HGD_HUMAN & 6.54 & 50625 & 25 & 58 \\
\hline 41. & 700 & Q9UQ35 & $\begin{array}{l}\text { Serine/arginine repetitive matrix } \\
\text { protein } 2\end{array}$ & SRRM2_HUMAN & 12.05 & 300179 & 11 & 72 \\
\hline 42. & 753 & P02753 & Retinol-binding protein 4 & RET4_HUMAN & 5.76 & 23337 & 61 & 93 \\
\hline
\end{tabular}

a Spot number.

${ }^{\mathrm{b}}$ Protein accession number for SWISSPROT Database.

c Theoretical isoelectric point.

${ }^{\mathrm{d}}$ Theoretical relative mass.

e MASCOT score 
Table S3: Biochemical parameters of the study subjects at baseline and after L-thyroxine therapy

\begin{tabular}{|l|c|c|c|}
\hline & Hypothyroid & Euthyroid & $p$-value \\
\hline $\mathrm{N}$ & $9(6 \mathrm{f})$ & $9(6 \mathrm{f})$ & \\
\hline Age (years) & $39.3 \pm 12.9$ & $39.3 \pm 12.9$ & \\
\hline Fasting glucose (mmol/L) & $5.3 \pm 0.4$ & $5.0 \pm 0.5$ & 0.078 \\
\hline Urea (mmol/L) & $4.7 \pm 0.7$ & $4.6 \pm 0.9$ & 0.392 \\
\hline Creatinine (mmol/L) & $72.5 \pm 13.1$ & $76.1 \pm 23.3$ & 0.404 \\
\hline Aspartate transaminase (IU/L) & $33.4 \pm 6.6$ & $37.5 \pm 9.0$ & 0.407 \\
\hline Alanine transaminase (IU/L) & $18.0 \pm 5.8$ & $17.4 \pm 3.9$ & 0.169 \\
\hline Alkaline phosphatase (IU/L) & $94.9 \pm 25.9$ & $96.8 \pm 31.2$ & 0.133 \\
\hline FT4 (pmol/L) & $8.3 \pm 5.5$ & $18.8 \pm 3.7$ & 0.000 \\
\hline TSH (mIU/l) & $33.9 \pm 22.1$ & $1.6 \pm 0.9$ & 0.000 \\
\hline Total Cholesterol (mmol/L) & $4.6 \pm 0.6$ & $4.8 \pm 0.7$ & 0.193 \\
\hline Triglycerides (mmol/L) & $1.2 \pm 0.3$ & $1.4 \pm 0.3$ & 0.184 \\
\hline LDL cholesterol (mmol/L) & $2.9 \pm 0.8$ & $3.0 \pm 0.6$ & 0.373 \\
\hline HDL cholesterol (mmol/L) & $1.2 \pm 0.4$ & $1.0 \pm 0.3$ & 0.087 \\
\hline
\end{tabular}


Table S4: Identified proteins, with changes in abundance of significantly differentially abundant proteins between hypothyroid and euthyroid states in urine samples. Table 2 shows values for the average ratio between the two states, with their corresponding levels of fold changes and one-way ANOVA (p-value < 0.05) using 2D-DIGE. [Analysis type: MALDI-TOF; database: SwissProt; taxonomy: Homo sapiens].

\begin{tabular}{|c|c|c|c|c|c|c|}
\hline 376 & P02768 & Serum albumin & ALBU_HUMAN & 0.009 & 1.9 & UP \\
\hline 700 & 075409 & Huntingtin-interacting protein $\mathrm{M}$ & HYPM_HUMAN & 0.002 & 3.3 & UP \\
\hline 117 & P02787 & Serotransferrin & TRFE_HUMAN & 0.044 & 4.5 & UP \\
\hline 742 & P21281 & $\begin{array}{l}\text { V-type proton ATPase subunit B, brain } \\
\text { isoform }\end{array}$ & VATB2_HUMAN & 0.006 & 2.3 & UP \\
\hline
\end{tabular}




\begin{tabular}{|c|c|c|c|c|c|c|}
\hline 743 & 095395 & $\begin{array}{l}\text { Beta-1,3-galactosyl-O-glycosyl- } \\
\text { glycoprotein beta-1,6-N- } \\
\text { acetylglucosaminyltransferase } 3\end{array}$ & GCNT3_HUMAN & 0.028 & 2.8 & UP \\
\hline 735 & Q5T197 & E3 ubiquitin-protein ligase DCST1 & DCST1_HUMAN & 0.014 & 2.4 & UP \\
\hline 750 & P07339 & Cathepsin D & CATD_HUMAN & 0.026 & 2.5 & UP \\
\hline 819 & Q8N806 & Putative E3 ubiquitin-protein ligase UBR7 & UBR7_HUMAN & 0.008 & 1.9 & UP \\
\hline 509 & P13645 & Keratin, type I cytoskeletal 10 & K1C10_HUMAN & 0.016 & 1.5 & UP \\
\hline 608 & Q8N7C3 & $\begin{array}{c}\text { Probable E3 ubiquitin-protein ligase } \\
\text { TRIML2 }\end{array}$ & TRIMM_HUMAN & 0.036 & 1.5 & UP \\
\hline 728 & Q6FIF0 & AN1-type zinc finger protein 6 & ZFAN6_HUMAN & 0.038 & 2.1 & UP \\
\hline 828 & Q6FIF0 & AN1-type zinc finger protein 6 & ZFAN6_HUMAN & 0.039 & 1.8 & UP \\
\hline 698 & P13647 & Keratin, type II cytoskeletal 5 & K2C5_HUMAN & 0.044 & 1.6 & UP \\
\hline 734 & P02760 & Protein AMBP & AMBP_HUMAN & 0.021 & 2.8 & UP \\
\hline
\end{tabular}




\begin{tabular}{|c|c|c|c|c|c|c|}
\hline 702 & Q12907 & $\begin{array}{l}\text { Vesicular integral-membrane protein } \\
\qquad \text { VIP36 }\end{array}$ & LMAN2_HUMAN & 0.018 & 2.2 & UP \\
\hline 498 & P01042 & Kininogen-1 & KNG1_HUMAN & 0.0015 & 1.8 & UP \\
\hline 578 & P02768 & Serum albumin & ALBU_HUMAN & 0.009 & 1.6 & UP \\
\hline 918 & Q8IZU9 & Kin of IRRE-like protein 3 & KIRR3_HUMAN & 0.014 & -3 & DOWN \\
\hline 644 & Q96CD2 & $\begin{array}{c}\text { Phosphopantothenoylcysteine } \\
\text { decarboxylase }\end{array}$ & COAC_HUMAN & 0.021 & -2.3 & DOWN \\
\hline 110 & Q8NFX7 & Syntaxin-binding protein 6 & STXB6_HUMAN & 0.042 & -1.7 & DOWN \\
\hline 481 & 095395 & $\begin{array}{l}\text { Beta-1,3-galactosyl-0-glycosyl- } \\
\text { glycoprotein beta-1,6-N- } \\
\text { acetylglucosaminyltransferase } 3\end{array}$ & GCNT3_HUMAN & 0.039 & 1.8 & UP \\
\hline
\end{tabular}




\begin{tabular}{|c|c|c|c|c|c|c|}
\hline 450 & 095395 & $\begin{array}{l}\text { Beta-1,3-galactosyl-O-glycosyl- } \\
\text { glycoprotein beta-1,6-N- } \\
\text { acetylglucosaminyltransferase } 3\end{array}$ & GCNT3_HUMAN & 0.041 & 2.0 & UP \\
\hline 808 & Q9HBZ2 & $\begin{array}{l}\text { Aryl hydrocarbon receptor nuclear } \\
\text { translocator } 2\end{array}$ & ARNT2_HUMAN & 0.04 & -2.1 & DOWN \\
\hline 503 & P01112 & GTPase HRas & RASH_HUMAN & 0.05 & -2.2 & DOWN \\
\hline 170 & P02768 & Serum albumin & ALBU_HUMAN & 0.049 & 3 & UP \\
\hline 655 & Q8NF91 & Nesprin-1 & SYNE1_HUMAN & 0.04 & -3.4 & DOWN \\
\hline 648 & Q9UQ35 & $\begin{array}{l}\text { Serine/arginine repetitive matrix protein } \\
\qquad 2\end{array}$ & SRRM2_HUMAN & 0.006 & -2.6 & DOWN \\
\hline 435 & Q8NF91 & Nesprin-1 & SYNE1_HUMAN & 0.010 & -2.5 & DOWN \\
\hline 493 & Q8NF91 & Nesprin-1 & SYNE1_HUMAN & 0.016 & -2.1 & DOWN \\
\hline 547 & Q6FIF0 & AN1-type zinc finger protein 6 & ZFAN6_HUMAN & 0.016 & -2.5 & DOWN \\
\hline
\end{tabular}




\begin{tabular}{|c|c|c|c|c|c|c|}
\hline 752 & Q96PF1 & $\begin{array}{l}\text { Protein-glutamine gamma- } \\
\text { glutamyltransferase Z }\end{array}$ & TGM7_HUMAN & 0.022 & -3.9 & DOWN \\
\hline 105 & A6NJ69 & IgA-inducing protein homolog & IGIP_HUMAN & 0.023 & -2.1 & DOWN \\
\hline 104 & Q9UP79 & $\begin{array}{l}\text { A disintegrin and metalloproteinase with } \\
\text { thrombospondin motifs } 8\end{array}$ & ATS8_HUMAN & 0.042 & -1.9 & DOWN \\
\hline 730 & 015212 & Prefoldin subunit 6 & PFD6_HUMAN & 0.030 & -2.1 & DOWN \\
\hline 756 & Q96KA5 & $\begin{array}{c}\text { Cleft lip and palate transmembrane } \\
\text { protein 1-like protein }\end{array}$ & CLP1L_HUMAN & 0.021 & 1.7 & UP \\
\hline 726 & P02760 & Protein AMBP & AMBP_HUMAN & 0.022 & 2.4 & UP \\
\hline 718 & A8K0R7 & Zinc finger protein 839 & ZN839_HUMAN & 0.026 & 2.5 & UP \\
\hline 741 & P02760 & Protein AMBP & AMBP_HUMAN & 0.052 & 2.1 & UP \\
\hline 802 & Q93099 & Homogentisate 1,2-dioxygenase & HGD_HUMAN & 0.053 & 1.6 & UP \\
\hline 700 & Q13438 & Protein OS-9 & OS9_HUMAN & 0.058 & 1.9 & UP \\
\hline
\end{tabular}


${ }^{a}$ Spot number.

b Protein accession number for SWISSPROT Database.

${ }^{c}$ Protein expression between hypothyroid and euthyroid states. 